\title{
An unusual face-on spiral in the wind of the M-type AGB star EP Aquarii (Corrigendum)
}

\author{
Ward Homan ${ }^{1}$, Anita Richards ${ }^{2}$, Leen Decin ${ }^{1,3}$, Alex de Koter ${ }^{1,5}$, and Pierre Kervella ${ }^{4}$ \\ ${ }^{1}$ Institute of Astronomy, KU Leuven, Celestijnenlaan 200D B2401, 3001 Leuven, Belgium \\ e-mail: ward.homan@kuleuven. be \\ 2 JBCA, Department Physics and Astronomy, University of Manchester, Manchester M13 9PL, UK \\ ${ }^{3}$ School of Chemistry, University of Leeds, Leeds LS2 9JT, UK \\ ${ }^{4}$ LESIA (CNRS UMR 8109), Observatoire de Paris, PSL, CNRS, UPMC, Univ. Paris Diderot, France \\ 5 Sterrenkundig Instituut “Anton Pannekoek", Science Park 904, 1098 XH Amsterdam, The Netherlands
}

A\&A, 616, A34 (2018), https://doi .org/10.1051/0004-6361/201832834

Key words. radiative transfer - stars: AGB and post-AGB - circumstellar matter - submillimeter: stars - molecular data errata, addenda

It has been brought to our attention that an error was made in the measurement of the continuum flux density (Sect. 3.1. and Fig. 1 in the original paper). The total continuum flux density within the $3 \sigma$ contour was erroneously calculated as $1.33 \mathrm{Jy}$ (being the total sum of the pixel intensities within this region), while in fact it is only $1.86 \mathrm{mJy}$. This implies that the deduction of the total dust mass that resides within this region $\left(\sim 5 \times 10^{-6} M_{\odot}\right)$ was false. However, we cannot correct this estimate by performing the same analysis to the updated flux measurement because our estimate of the stellar continuum flux contribution of $2.27 \times 10^{-2} \mathrm{Jy}$ exceeds the updated value. Hence, we hereby retract all statements regarding the nature of the dust within the $3 \sigma$ contour made in the second paragraph of Sect. 3.1. in the original manuscript. With the current values, we can at this point only state that either the stellar brightness and temperature (Winters et al. 2007) need to be better measured, or that the dust near the star is optically thick and prevents part of the stellar flux to penetrate all the way through to the observer.

Acknowledgements. We would like to sincerely thank Prof. A Zijlstra for bringing the abovementioned issue to our attention.

\section{Reference}

Winters, J. M., Le Bertre, T., Pety, J., \& Neri, R. 2007, A\&A, 475, 559 